\title{
Generalized Thermoelasticity for an Infinite Solid Cylinder*
}

\author{
Toshio FURUKAWA**, Naotake NODA*** \\ and Fumihiro ASHIDA****
}

\begin{abstract}
One-dimensional, generalized thermoelasticity is presented based on Lord and Shulman's theory and Green and Lindsay's theory. The former theory involves one relaxation time of the thermoelastic process and the latter involves two relaxation times. These theories have been developed in an attempt to eliminate the paradox of the infinite velocity of thermoelastic propagation inherent in the classical dynamically coupled theory. A formulation of generalized thermoelasticity which combines both generalized theories is derived. The generalized thermoelastic problems in an infinite solid cylinder are analyzed by means of a Laplace transform technique. The numerical calculations for displacement, temperature and stress under the generalized formulation are carried out and compared with the results under the classical formulation.
\end{abstract}

Key Words : Elasticity, Thermal Stress, Generalized Thermoelasticity, Lord and Shulman's Theory, Green and Lindsay's Theory, Relaxation Time, Infinite Solid Cylinder, Short-Time Solution

\section{Introduction}

When an isotropic and homogeneous elastic solid is subjected to a thermal disturbance, the effect is instantaneous at a location distant from the source in the classical linear thermoelastic theory. This means that the thermal wave propagates at infinite velocity which is a physically unreasonable result. Two gener. alized thermoelastic theories are proposed to eliminate the paradox and correct the classical theory on the assumption that a thermal wave propagates at finite velocity. These theories are by Lord and Shulman ${ }^{(1)}$ and Green and Lindsay ${ }^{(2)}$. The former is a thermoelastic theory including the finite velocity of a

* Received 22nd November, 1990. Paper No.89-0453A

** Department of Mechanical and System Engineering, Kyoto Institute of Technology, Goshokaido-cho, Matsugasaki, Sakyo-ku, Kyoto 606, Japan

*** Department of Mechanical Engineering, Shizuoka University, 5-1 Johoku 3-chome, Hamamatsu 432, Japan

**** Department of Mechanical Engineering, Tsuyama National College of Technology, 624-1 Numa, Tsuyama 708, Japan thermal wave by correcting the Fourier thermal conduction law and by introducing one relaxation time of the thermoelastic process. The latter is a theory that considers the finite velocity of a thermal wave by correcting the energy equation and DuhamelNeumann relation, and by introducing two relaxation times of the thermoelastic process.

We dealt with the one-dimensional generalized thermoelasticity for an infinite solid with a circular cylindrical hole based on Lord and Shulman's theory ${ }^{(3)}$ and Green and Lindsay's theory ${ }^{(4)}$ respectively. These results showed that the latter analytical procedure was almost the same as the former one. Therefore, the fundamental equations common to the two theories are first introduced for a solid cylinder possessing finite domain and an approximate analysis for short periods of time is carried out by the use of these equations. The collected treatment is possible for the problems for which individual investigations have been conducted. The numerical results of displacement, temperature and thermal stress distributions based on the two theories are examined. 


\section{Analysis}

We consider the one-dimensional generalized thermoelasticity of an infinite solid cylinder whose radius is $a$. The boundary condition is that a constant heat flux $q_{0}$ is flowing into the cylinder from the surface, but the displacement at the surface is constrained.

The radial strain $\varepsilon_{r r}$ and the hoop strain $\varepsilon_{\theta \theta}$ are given by

$$
\varepsilon_{r r}=u, r, \varepsilon_{\theta \theta}=\frac{u}{r},
$$

where $u$ is a radial displacement, and the commas denote differentiation with respect to the variable following.

At present, two different theories exist together in the generalized thermoelasticity. They are Lord and Shulman's theory and Green and Lindsay's theory. The former theory involves one relaxation time of the thermoelastic process and the latter involves two relaxation times. The fundamental equations of each theory are different, but the introduction of Kronecker's delta $\delta_{1 k}$ makes common fundamental equations possible, where the subscript $k$ denotes the number of the relaxation time.

The stress-displacement relations are

$$
\begin{gathered}
{\left[\begin{array}{c}
\sigma_{r r} \\
\sigma_{\theta \theta}
\end{array}\right]=2 \mu\left[\begin{array}{l}
u, r \\
u / r
\end{array}\right]+\lambda\left(u, r+\frac{u}{r}\right)} \\
-(3 \lambda+2 \mu) \alpha\left(T+t_{1} T_{, t}\right),
\end{gathered}
$$

where $\sigma_{r r}$ and $\sigma_{\theta \theta}$ are the radial stress and the hoop stress, respectively, $\lambda$ and $\mu$ are Lame's constants, $\alpha$ is a coefficient of linear thermal expansion, $T$ is temperature and $t_{1}$ is relaxation time.

The equation of motion presented by the displacement component is

$$
u, r r+\frac{1}{r} u, r-\frac{u}{r^{2}}-\zeta \alpha\left(T+t_{1} T,_{t}\right)_{, r}=\frac{1}{v_{e}^{2}} u, t t,
$$

where $v_{e}$ is the propagation velocity of a longitudinal wave, and $\zeta=(1+\nu) /(1-\nu)$ with Poisson's ratio $\nu$.

The one-dimensional heat conduction equation with respect to the generalized thermoelasticity is given by

$$
\begin{aligned}
& x\left(T, r r+\frac{1}{r} T, r\right)-\left(T+t_{0} T, t\right), t \\
& \quad=\frac{\delta}{\zeta \alpha}\left[u, r+\frac{u}{r}+\delta_{1 k} t_{0}\left(u, r+\frac{u}{r}\right), t\right], t,
\end{aligned}
$$

where $x$ is thermal diffusivity, $t_{0}$ is relaxation time, and $\delta$ is a thermomechanical coupling parameter defined by

$$
\delta=\frac{(3 \lambda+2 \mu)^{2} \alpha^{2} \tilde{T}}{(\lambda+2 \mu) \tilde{\rho} c_{v}} .
$$

Here, $\tilde{T}$ is an absolute reference temperature, $\tilde{\rho}$ is density and $c_{v}$ is a specific heat at constant volume.
The above fundamental equations correspond to the individual theoretical equations which follow:

(1) Lord and Shulman's theory

$$
\delta_{1 k}=1, t_{1}=0
$$

(2) Green and Lindsay's theory

$$
\delta_{1 k}=0, t_{1} \geqq t_{0} \geqq 0 .
$$

The next relation is introduced from Eqs. $(6 \cdot a)$ and $(6 \cdot b)$.

$$
\delta_{1 k} t_{1}=0
$$

We introduce the following dimensionless quantities:

$$
\begin{gathered}
\rho=\frac{r}{a}, \tau=\frac{\chi t}{a^{2}}, \theta=\frac{T}{T_{0}}, \beta=\frac{\chi}{v_{e} a} \\
{\left[\frac{\widehat{\rho \rho}}{\hat{\theta \theta}}\right]=\frac{1}{(\lambda+2 \mu) \alpha T_{0}}\left[\begin{array}{l}
\sigma_{r r} \\
\sigma_{\theta \theta}
\end{array}\right]} \\
\left.U=\frac{u}{a \alpha T_{0}}, \tau_{0}=\frac{\chi t_{0}}{a^{2}}, \tau_{1}=\frac{\chi t_{1}}{a^{2}}\right],
\end{gathered}
$$

where $T_{0}$ is a reference temperature. When we make the dimensionless forms of Eqs. ( 2$) \sim(4)$ by the use of Eq. ( 8 ) and perform the Laplace transform on these equations under the initial condition

$$
\tau=0 ; U=U, \tau=0, \theta=\theta, \tau=0,
$$

the next three equations are obtained :

$$
\begin{aligned}
& {\left[\begin{array}{c}
{\left[\frac{\rho \rho^{*}}{\theta \theta^{*}}\right]} \\
\quad-\zeta\left(1+\tau_{1} p\right) \theta^{*} \\
\eta
\end{array}\right]\left(U_{, \rho}^{*}+\frac{U^{*}}{\rho}\right) \pm(\eta-1) \frac{U^{*}}{\rho}} \\
& U_{, \rho \rho}^{*}+\frac{1}{\rho} U_{, \rho}^{*}-\left(\beta^{2} p^{2}+\frac{1}{\rho^{2}}\right) U^{*} \\
& \quad-\zeta\left(1+\tau_{1} p\right) \theta_{, \rho}^{*}=0 \\
& \theta_{, \rho \rho}^{*}+\frac{1}{\rho} \theta_{, \rho}^{*}-\left(1+\tau_{0} p\right) p \theta^{*} \\
& \quad=\frac{\delta}{\zeta} p\left(1+\delta_{1 k} \tau_{0} p\right)\left(U_{, \rho}^{*}+\frac{U^{*}}{\rho}\right),
\end{aligned}
$$

where the superscripted asterisk denotes the Laplace transform, $p$ is a transformation parameter, and $\eta=$ $\nu /(1-\nu)$.

The combination of Eqs. (11) and (12) leads to the following equations of displacement and temperature:

$$
\begin{aligned}
& U^{*}=\zeta\left(1+\tau_{1} p\right)\left[A_{1} \xi_{1} I_{1}\left(\xi_{1} \rho\right)+A_{2} \xi_{2} I_{1}\left(\xi_{2} \rho\right)\right] \\
& \theta^{*}=\left(\xi_{1}^{2}-\beta^{2} p^{2}\right) A_{1} I_{0}\left(\xi_{1} \rho\right)+\left(\xi_{2}^{2}-\beta^{2} p^{2}\right) A_{2} I_{0}\left(\xi_{2} \rho\right),
\end{aligned}
$$

where $A_{1}$ and $A_{2}$ are integral constants, $I_{i}(\quad)$ is a modified Bessel function of order $i$ of the first kind, and $\xi_{1}$ and $\xi_{2}$ are the positive roots of the following transcendental equation : where

$$
\xi^{4}-\left(B_{0} p+B_{1}\right) p \xi^{2}+\left(1+\tau_{0} p\right) \beta^{2} p^{3}=0,
$$

$$
B_{0}=\beta^{2}+\tau_{0}+\delta\left(\tau_{1}+\delta_{1 k} \tau_{0}\right), B_{1}=1+\delta .
$$

We obtain from Eq. (15),

$$
\begin{aligned}
\xi_{i}^{2} & =\frac{p}{2}\left\{B_{0} p+B_{1}+(-1)^{i-1}\left[\left(B_{0}^{2}-4 \tau_{0} \beta^{2}\right) p^{2}\right.\right. \\
& \left.\left.+2\left(B_{0} B_{1}-2 \beta^{2}\right) p+B_{1}^{2}\right]^{1 / 2}\right\}, \quad(i=1,2) .
\end{aligned}
$$

We substitute Eqs.(13) and (14) into Eq.(10) and obtain 


$$
\begin{aligned}
& {\left[\begin{array}{c}
{\widehat{\rho \rho^{*}}}_{\theta \theta^{*}}^{*}
\end{array}\right]=\left[\begin{array}{c}
1 \\
\eta
\end{array}\right] \zeta\left(1+\tau_{1} p\right)\left[A_{1} \xi_{1}^{2} I_{0}\left(\xi_{1} \rho\right)+A_{2} \xi_{2}^{2} I_{0}\left(\xi_{2} \rho\right)\right]} \\
& \pm(\eta-1) \frac{U^{*}}{\rho}-\zeta\left(1+\tau_{1} p\right) \theta^{*} .
\end{aligned}
$$

We will seek the solution for short periods of time. As the parameter $p$ is large enough in this case, Eq. (17) is modified as follows :

$$
\begin{aligned}
\xi_{i}^{2} & =\frac{p^{2}}{2}\left[B_{0}+(-1)^{i-1} C_{0}+\frac{B_{1}+(-1)^{i-1} C_{1}}{p}\right. \\
& \left.+\frac{(-1)^{i-1} C_{2}}{p^{2}}+\frac{(-1)^{i-1} C_{3}}{p^{3}}\right],(i=1,2),
\end{aligned}
$$

where

$$
\left.\begin{array}{l}
C_{0}=\left(B_{0}^{2}-4 \tau_{0} \beta^{2}\right)^{1 / 2}, C_{1}=\frac{B_{0} B_{1}-2 \beta^{2}}{C_{0}} \\
C_{2}=\frac{2 \beta^{2}\left(B_{0} B_{1}-\tau_{0} B_{1}^{2}-\beta^{2}\right)}{C_{0}^{3}}, C_{3}=-\frac{C_{1} C_{2}}{C_{0}}
\end{array}\right\},
$$

and the number of the series-expanding terms is limited to four. Then we obtain

$$
\begin{aligned}
& \frac{I_{j}\left(\xi_{i} \rho\right)}{I_{1}\left(\xi_{i}\right)}=\frac{1}{\sqrt{\rho}} e^{-\xi_{i}(1-\rho)}\left(1+\frac{\rho_{j 1}}{\xi_{i}}+\frac{\rho_{j 2}}{\xi_{i}^{2}}\right), \\
& \quad(i=1,2, j=0,1),
\end{aligned}
$$

where

$$
\left.\begin{array}{l}
\rho_{01}=\frac{1}{8}\left(3+\frac{1}{\rho}\right), \rho_{02}=\frac{3}{128}\left(11+\frac{2}{\rho}+\frac{3}{\rho^{2}}\right) \\
\rho_{11}=\frac{3}{8}\left(1-\frac{1}{\rho}\right), \rho_{12}=\frac{3}{128}\left(11-\frac{6}{\rho}-\frac{5}{\rho^{2}}\right)
\end{array}\right\},
$$

and the number of the series-expanding terms is limited to three.

After this, we examine the case in which the value of the relaxation time $\tau_{0}$ is not zero.

Equation (19) is modified into the following forms :

$$
\xi_{i}^{2}=a_{i 0}^{2} p^{2}\left(1+\frac{a_{i 1}}{p}+\frac{a_{i 2}}{p^{2}}\right), \quad(i=1,2)
$$

where

$$
\left.\begin{array}{l}
a_{i 0}=\left\{\frac{1}{2}\left[B_{0}+(-1)^{i-1} C_{0}\right]\right\}^{1 / 2} \\
\left.a_{i 1}=\frac{B_{1}+(-1)^{i-1} C_{1}}{B_{0}+(-1)^{i-1} C_{0}}, \quad(i=1,2)\right\} \\
a_{i 2}=\frac{(-1)^{i-1} C_{2}}{B_{0}+(-1)^{i-1} C_{0}}
\end{array}\right\}
$$

From Eq. (23), we obtain

$$
\xi_{i}=a_{i 0} p\left(1+\frac{a_{i 3}}{p}+\frac{a_{i 4}}{p^{2}}\right), \quad(i=1,2)
$$

$$
A_{i}=\frac{(-1)^{i-1} Q_{0}}{p\left(\xi_{1}^{2}-\xi_{2}^{2}\right) \xi_{i} I_{1}\left(\xi_{i}\right)}, \quad(i=1,2)
$$

Introducing Eq. (32) into Eqs. (13),(14) and (18), we obtain the solutions in the Laplace domain. Performing the inverse Laplace transform, we obtain the following equations for displacement, temperature, and thermal stress distributions :

$$
\begin{aligned}
& U=\frac{\zeta Q_{0}}{C_{0}}\left[g_{11}\left(\tau_{a 1}\right)-g_{12}\left(\tau_{a 2}\right)\right] \\
& \theta=\frac{Q_{0}}{2 C_{0}}\left[g_{21}\left(\tau_{a 1}\right)-g_{22}\left(\tau_{a 2}\right)\right] \\
& {\left[\frac{\widehat{\rho \rho}}{\partial \theta}\right]=\left[\begin{array}{l}
1 \\
\eta
\end{array}\right] \frac{\zeta Q_{0}}{C_{0}}\left[g_{31}\left(\tau_{a 1}\right)-g_{32}\left(\tau_{a 2}\right)\right] \pm(\eta-1) \frac{U}{\rho}-\frac{\zeta Q_{0}}{2 C_{0}}\left[g_{41}\left(\tau_{a 1}\right)-g_{42}\left(\tau_{a 2}\right)\right],}
\end{aligned}
$$


where

$$
\begin{aligned}
\tau_{a i}=\tau & -a_{i 0}(1-\rho), \quad(i=1,2) \\
g_{1 i}\left(\tau_{a i}\right)= & X_{i i} \tau_{a i}\left[\tau_{1}+\frac{1}{2} \tau_{a i}+\frac{1}{2} \tau_{a i}\left(\tau_{1}+\frac{1}{3} \tau_{a i}\right) a_{\rho i 6}\right. \\
& \left.+\frac{1}{6} \tau_{a i}^{2}\left(\tau_{1}+\frac{1}{4} \tau_{a i}\right)\left(a_{\rho i 7}-C_{10} a_{\rho i 6}\right)\right] H\left(\tau_{a i}\right), \quad(i=1,2) \\
g_{2 i}\left(\tau_{a i}\right)= & \frac{X_{i}}{a_{i 0}} \tau_{a i}\left[a_{i 5}+\frac{1}{2} \tau_{a i}\left[a_{i 5}\left(a_{\rho i 4}-a_{i 3}\right)+a_{i 6}\right]+\frac{1}{6}\left\{a_{i 5} a_{\rho i 5}\right.\right. \\
& \left.\left.-\left[a_{i 5}\left(a_{i 3}+C_{10}\right)-a_{i 6}\right] a_{\rho i 4}+a_{i 5}\left(a_{i 3}^{2}-a_{i 4}\right)-a_{i 3} a_{i 6}+a_{i 7}\right\} \tau_{a i}^{2}\right], \quad(i=1,2) \\
g_{3 i}\left(\tau_{a i}\right)= & a_{i 0} X_{i}\left\{\tau_{1}+\tau_{a i}+\tau_{a i}\left(\tau_{1}+\frac{1}{2} \tau_{a i}\right)\left(a_{\rho i 4}+a_{i 3}\right)\right. \\
& \left.+\frac{1}{2} \tau_{a i}^{2}\left(\tau_{1}+\frac{1}{3} \tau_{a i}\right)\left[a_{\rho i 5}+\left(a_{i 3}-C_{10}\right) a_{\rho i 4}+a_{i 4}\right]\right\} H\left(\tau_{a i}\right), \quad(i=1,2) \\
g_{i 4}\left(\tau_{a i}\right)= & \frac{X_{i}}{a_{i 0}}\left[\left(\tau_{1}+\tau_{a i}\right) a_{\rho i 5}+\tau_{a i}\left(\tau_{1}+\frac{1}{2} \tau_{a i}\right)\left[a_{i 5}\left(a_{\rho i 4}-a_{i 3}\right)+a_{i 6}\right]\right. \\
& +\frac{1}{2} \tau_{a i}^{2}\left(\tau_{1}+\frac{1}{3} \tau_{a i}\right)\left\{a_{i 5} a_{\rho i 5}-\left[a_{i 5}\left(a_{i 3}+C_{10}\right)-a_{i 6}\right] a_{\rho i 4}\right. \\
& \left.+a_{i 5}\left(a_{i 3}^{2}-a_{i 4}\right)-a_{i 3} a_{i 6}+a_{i 7}\right], \quad(i=1,2) \\
a_{\rho i 4}= & a_{\rho i 0}-C_{10}, a_{\rho i 5}=a_{\rho i 1}-C_{20}, a_{\rho i 6}=a_{\rho i 2}-C_{10}, a_{\rho i 7}=a_{\rho i 3}-C_{20}, \quad(i=1,2) \\
C_{10}= & C_{1} / C_{0,}, C_{20}=C_{2} / C_{0 .}
\end{aligned}
$$

\section{Numerical Examples and Discussion}

Numerical examples are carried out under the following conditions used in the previous papers ${ }^{(3),(4)}$ : $Q_{0}=1, \beta=0.01, \nu=0.3, \delta=0.1, \tau=10^{-5}$

(Lord and Shulman's theory): $\tau_{0}=10^{-5} \sim 10^{-4}$ (Green and Lindsay's theory): $\tau_{0}=10^{-5}, \tau_{1}=10^{-5} \sim 10^{-3}$.

Figures 1 $\sim 4$ show the displacement, temperature, radial stress, and hoop stress distributions in the vicinity of the surface of the solid cylinder based on two generalized theories and the classical dynamically coupled theory. In these figures, the notations L-S $\left(\tau_{0}\right.$ $\left.=10^{-5}\right), \mathrm{G}-\mathrm{L}\left(\tau_{0}=\tau_{1}=10^{-5}\right)$ and $\mathrm{C}-\mathrm{D}\left(\tau_{0}=0\right)$ denote the

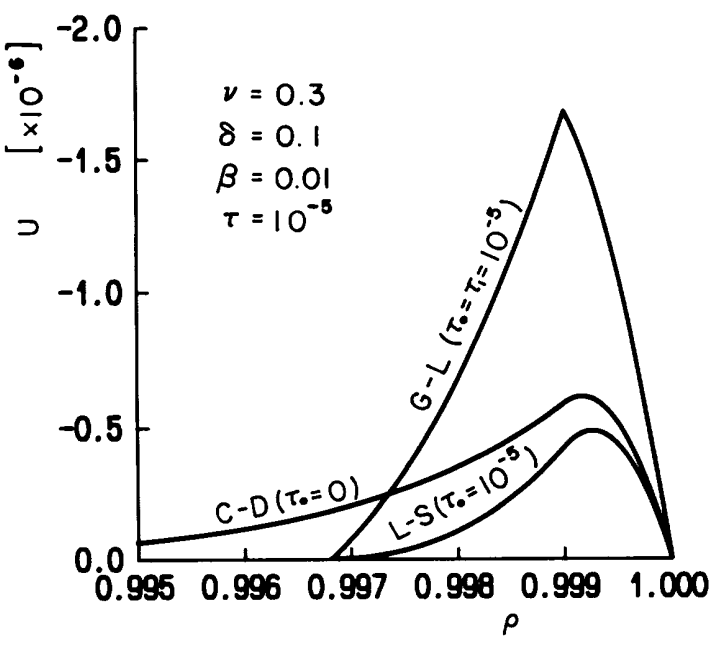

Fig. 1 Displacement distributions in the vicinity of the surface of the infinite solid cylinder (comparison between two theories) following. The first represents Lord and Shulman's theory for the case in which the dimensionless relaxation time $\tau_{0}$ is $10^{-5}$. The second is Green and Lindsay's theory for the case in which two dimensionless relaxation times $\tau_{0}$ and $\tau_{1}$ are both equal to $10^{-5}$. The last is the classical dynamically coupled theory implying that the dimensionless relaxation time $\tau_{0}$ is exactly zero.

The displacement of Lord and Shulman's theory shows smooth variation and is always smaller than that of the classical one. On the other hand, the displacement of Green and Lindsay's theory shows rapid variation at the stress wavefront and is larger than that of the classical one for the most part. Thus,

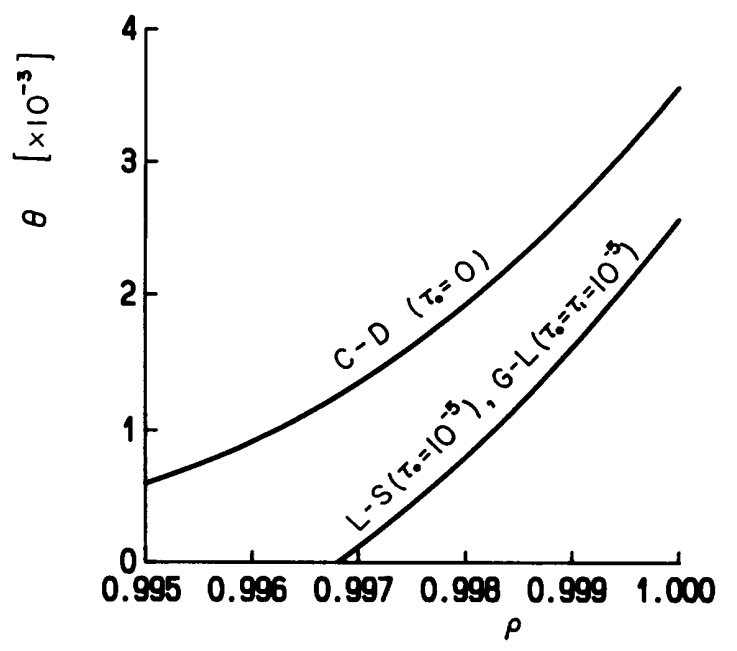

Fig. 2 Temperature distributions in the vicinity of the surface of the infinite solid cylinder (comparison between two theories) 


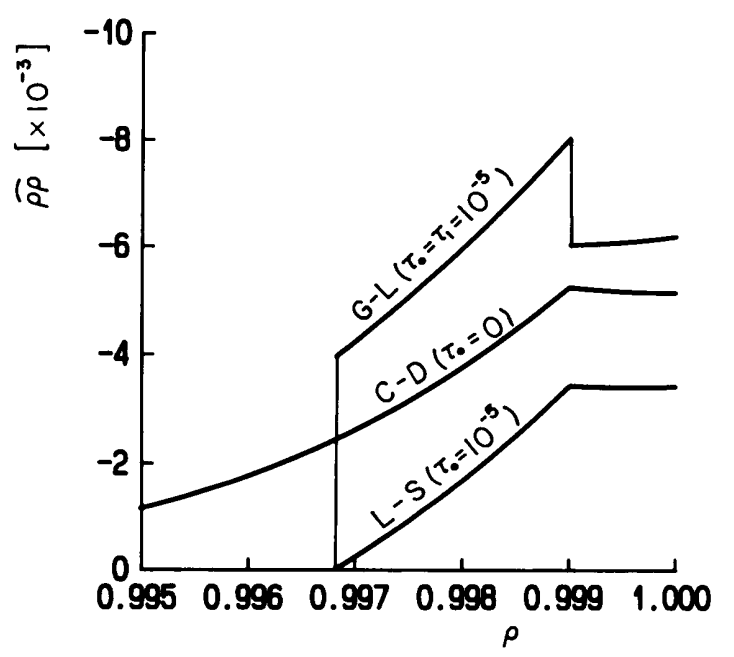

Fig. 3 Radial stress distributions in the vicinity of the surface of the infinite solid cylinder (comparison between two theories)

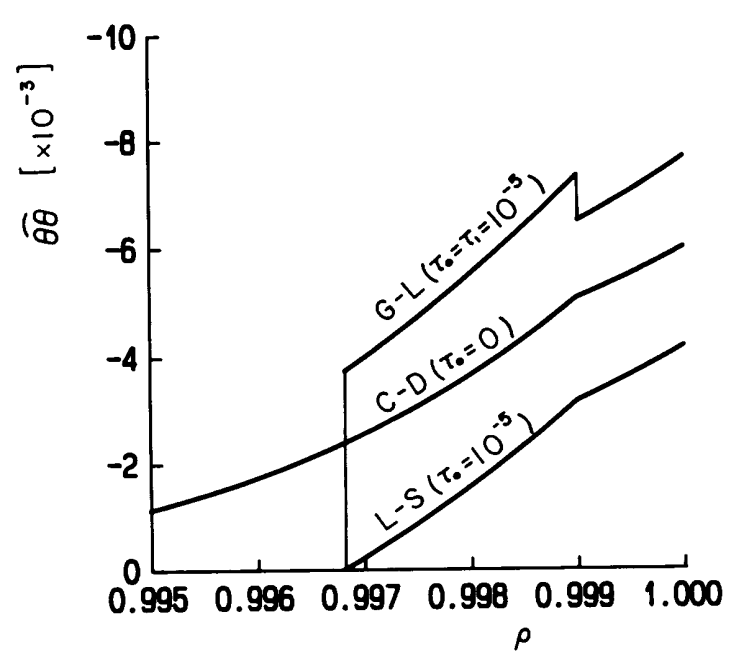

Fig. 4 Hoop stress distributions in the vicinity of the surface of the infinite solid cylinder (comparison between two theories)

the clear difference between the two generalized theories appears in these displacement distributions. However, the temperature distribution of Lord and Shulman's theory coincides with that of Green and Lindsay's theory. No difference between the two theories appears in these temperature distributions. The jumps of radial and hoop stresses of Green and Lindsay's theory occur at the stress and thermal wavefronts. No jump of radial and hoop stresses of Lord and Shulman's theory occurs. Hence, the clear difference between the two theories appears in these stress distributions, similarly to displacement distributions. As the consideration of relaxation time implies the finite speed of the thermal wave, the initial state is maintained at the region which the thermal wave has not arrived, and physical rationality is kept.

Figures $5 \sim 7$ show the displacement, temperature

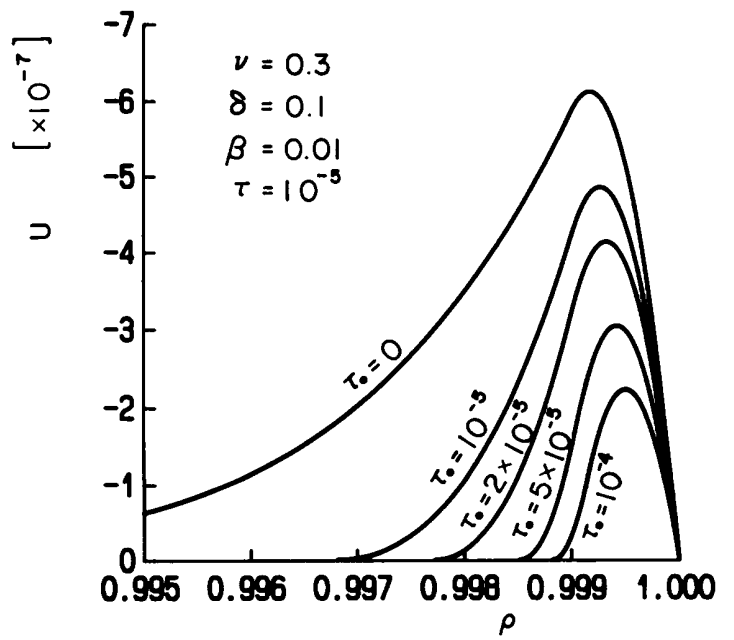

Fig. 5 Displacement distributions in the vicinity of the surface of the infinite solid cylinder based on Lord and Shulman's theory

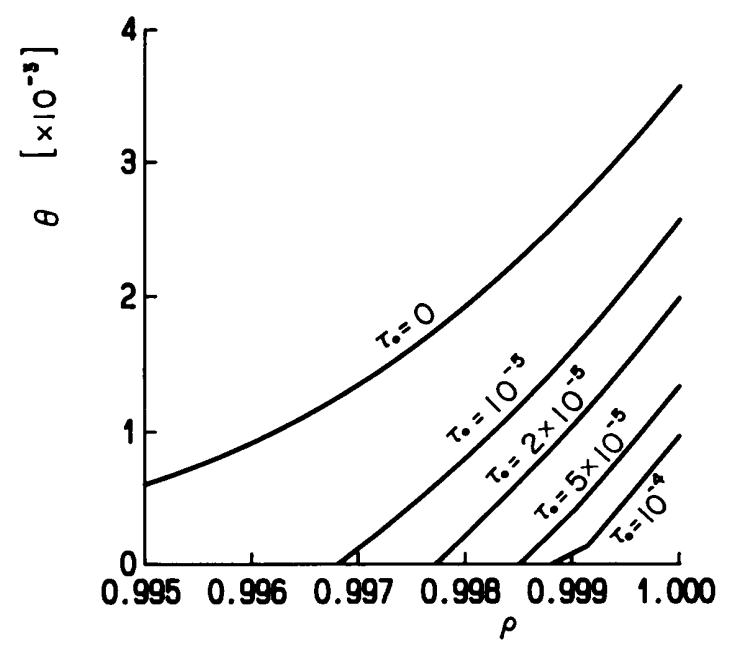

Fig. 6 Temperature distributions in the vicinity of the surface of the infinite solid cylinder based on Lord and Shulman's theory

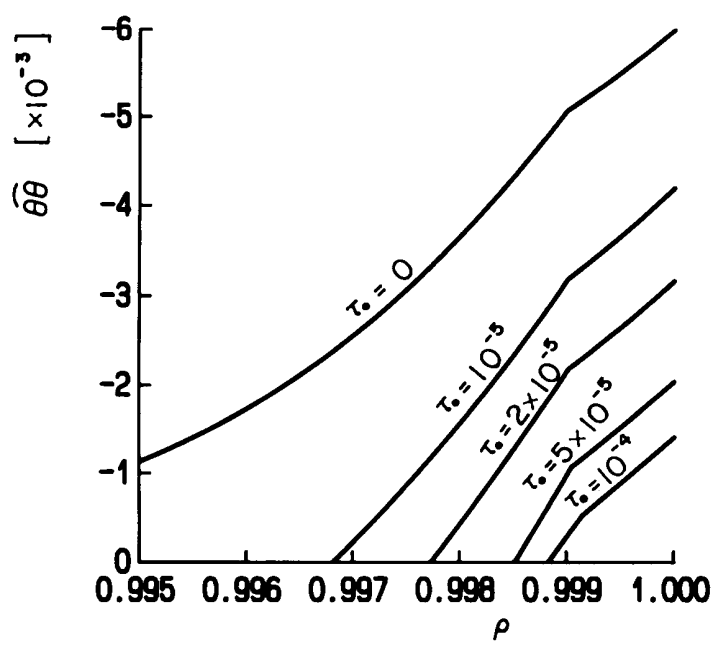

Fig. 7 Hoop stress distributions in the vicinity of the surface of the infinite solid cylinder based on Lord and Shulman's theory

Series I, Vol. 34, No. 3, 1991 


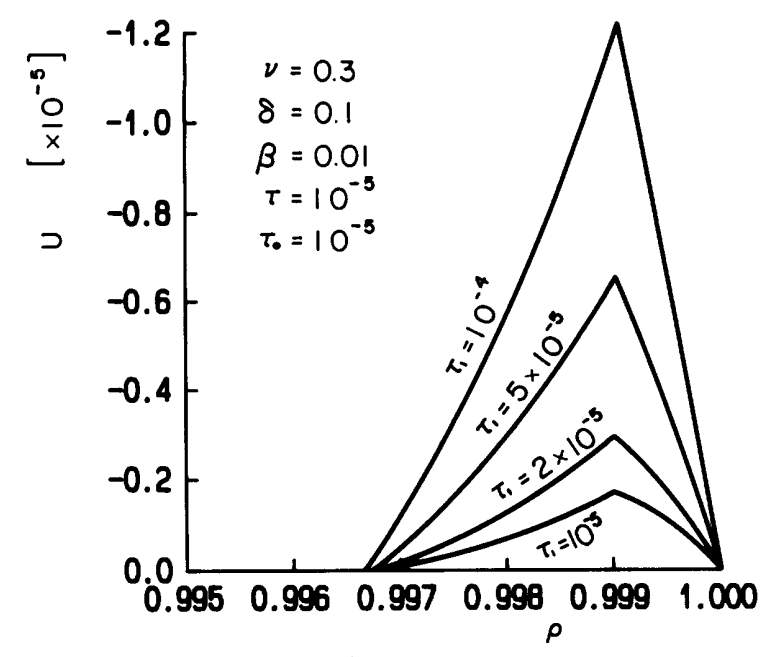

Fig. 8 Displacement distributions in the vicinity of the surface of the infinite solid cylinder based on Green and Lindsay's theory

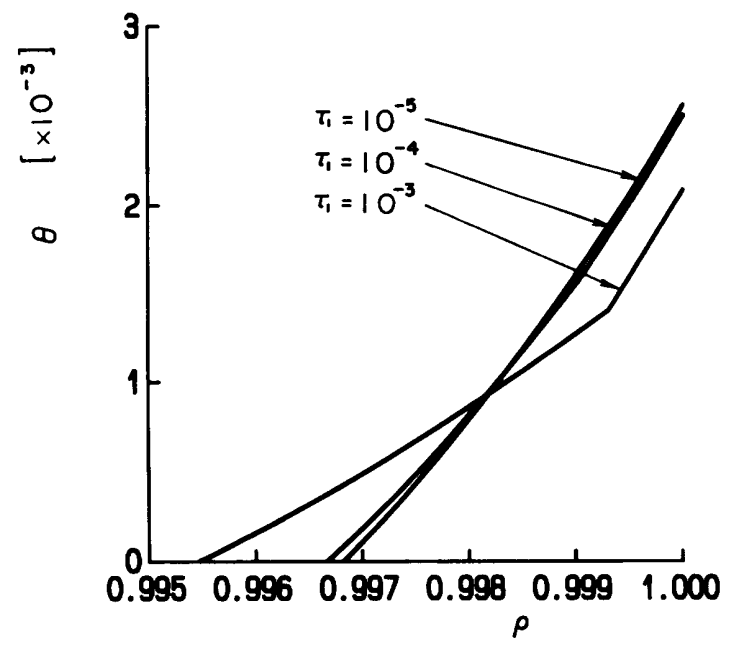

Fig. 9 Temperature distributions in the vicinity of the surface of the infinite solid cylinder based on Green and Lindsay's theory

and hoop stress distributions in the vicinity of the surface of the solid cylinder based on Lord and Shulman's theory. The displacement magnitudes increase gradually and approach the result based on the classical theory when the dimensionless relaxation time becomes small. The tendency of temperature distributions is similar to that of displacement. The rapid variations of hoop stress distributions occur at the stress wavefront.

Figures $8 \sim 10$ show the displacement, temperature and hoop stress distributions in the vicinity of the surface of the solid cylinder based on Green and Lindsay's theory. When dimensionless relaxation time $\tau_{1}$ becomes larger than $\tau_{0}$, the displacement becomes large. However, a clear difference does not appear in

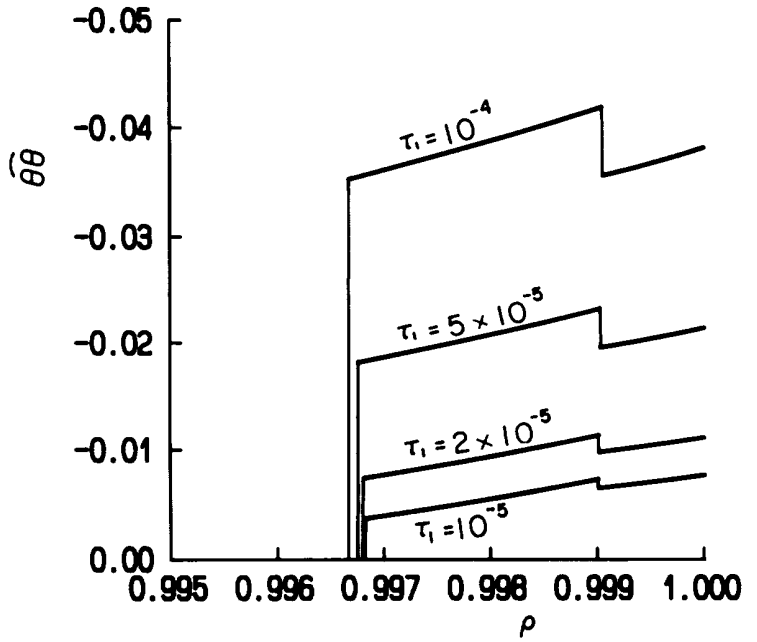

Fig. 10 Hoop stress distributions in the vicinity of the surface of the infinite solid cylinder based on Green and Lindsay's theory

the temperature distributions even when the difference between the two relaxation times is ten times. Therefore, the undistinguished difference between the two theories appears in the temperature distributions. Large dimensionless relaxation time $\tau_{1}$ leads to the large discontinuities of the hoop stress at the stress and thermal wavefronts.

An approximate analysis for short periods of time of one-dimensional generalized thermoelasticity for an infinite solid cylinder supplied with a constant heat flux is presented. The analyses based on the two theories have been performed separately in the past. We introduce the common fundamental equations in this paper. The use of these equations enables us to consider two theories at the same time. The numerical calculations show quite different tendencies between these theories.

\section{References}

(1) Lord, H. W. and Shulman, Y., A Generalized Dynamical Theory of Thermoelasticity, J. Mech. Phys. Solids, Vol.15(1967), p.299.

(2) Green, A. E. and Lindsay, K. A., Thermoelasticity, J. Elasticity, Vol.2, No.1 (1972), p.1.

(3) Furukawa, T., Noda, N. and Ashida, F., Generalized Thermoelasticity for an Infinite Body with a Circular Cylindrical Hole, Trans. Jpn. Soc. Mech. Eng., (in Japanese), Vol.54, No.506, (1988), p.1855.

(4) Furukawa, T., Noda, N. and Ashida, F., Generalized Thermoelasticity for an Infinite Body with a Circular Cylindrical Hole Based on Green and Lindsay's Theory, Trans. Jpn. Soc. Mech. Eng., (in Japanese), Vol.55, No.517, (1989), p.1998. 